\title{
CONSTRUYENDO LA SALUD PÚBLICA DEL FUTURO. PLANIFICACIÓN ESTRATÉGICA EN LA ESCUELA DE SALUD PÚBLICA DE LA UNIVERSIDAD DE CHILE
}

\section{Giorgio Solimano, Julia González ${ }^{1}$}

\begin{abstract}
RESUMEN
Este artículo presenta la experiencia de gestión académica 2000-2010 en la Escuela de Salud Pública (ESP) de la Universidad de Chile, en la cual se formuló un plan basado en una metodología de planificación estratégica participativa. Los tres ejes fueron generar un quehacer académico de excelencia, fortalecer la relación con el entorno y establecer una organización moderna al servicio del quehacer académico.

Se identifica un conjunto de logros destacando la formación de recursos humanos avanzados; el fortalecimiento del cuerpo académico, la ampliación de las relaciones con el entorno y la profesionalización de la gestión académica. Se identifican los factores que facilitaron estos logros, así como aquellos que dificultaron su implementación y estrategias diseñadas para superarlos. Se concluye que esta experiencia constituyó un efectivo aporte a la modernización de la ESP y al diseño, gestión y evaluación de políticas públicas en el ámbito académico.
\end{abstract}

Palabras clave: salud pública, planificación estratégica, gestión

\section{BUILDING THE PUBLIC HEALTH OF THE FUTURE: STRATEGIC PLANNING AT THE SCHOOL OF PUBLIC HEALTHOF UNIVERSITY OF CHILE}

\section{ABSTRACT}

This article presents the experience in academic management 2000-2010 at the School of Public Health of University of Chile developing a Plan based on a participatory methodology of strategic planning. The three objectives were to promote academic excellence, to strengthen relationships with other actors and to establish a modern organization that would serve the academic community.

Several achievements are identified, including advanced human resources training, strengthening of the academic body, expanded external relationships and professionalizing academic management. It identifies those factors facilitating and those restricting the implementation of the plan and strategies to overcome them. In conclusion, this experience was an important contribution to the modernization of the School and the design, management and evaluation of public policies in an academic setting.

Keywords: public health, strategic planning, management

1 Escuela de Salud Pública, Facultad de Medicina, Universidad de Chile. Santiago, Chile. Contacto: gsolimano@med.uchile.cl. 


\section{CONSTRUYENDO LA SALUD PÚBLICA DEL FUTURO. PLANIFICACIÓN ESTRATÉGICA EN LA ESCUELA DE SALUD PÚBLICA DE LA UNIVERSIDAD DE CHILE}

\section{Introducción}

La Escuela de Salud Pública, ESP, fundada en 1943, es depositaria de una historia de 67 años en el campo de la salud pública en Chile. En sus aulas se han formado la mayoría de los salubristas chilenos y un gran número de latinoamericanos. Forma parte de la Facultad de Medicina de la Universidad de Chile y su estructura organizacional es matricial (figura 1).

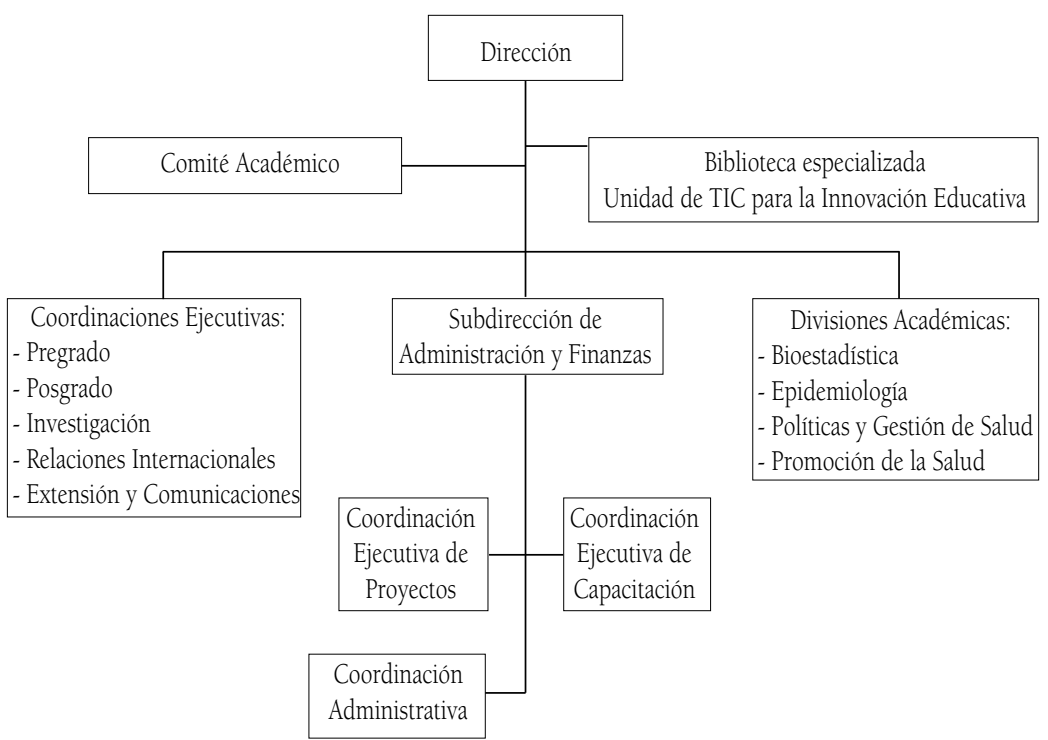

La salud colectiva o poblacional constituye el objetivo de la salud pública y el campo del quehacer de la ESP. En este contexto, la Escuela de Salud Pública ha definido su misión como "contribuir a mejorar la salud de la población en el ámbito nacional e internacional, 
mediante la búsqueda de la excelencia en la investigación, formación de recursos humanos, extensión y asistencia técnica".

Al inicio de la segunda década del siglo XXI enfrentamos un nuevo escenario en salud en el país y en el mundo, lo que unido a la naturaleza interdisciplinaria de la salud pública, determina que esta enfrente nuevos desafíos que obligan a la ESP a responder con un efectivo liderazgo. Con el fin de dar respuesta a estos nuevos desafíos, a contar del año 2000 la ESP inició un proceso de modernización e innovación, sustentado en un Plan Estratégico de Desarrollo, PED, orientado a mejorar la calidad del quehacer académico, satisfacer los requerimientos del entorno y fortalecer el perfeccionamiento y la participación del personal académico y no académico.

El objetivo de este trabajo es dar a conocer la experiencia de diseño, implementación y evaluación del Plan Estratégico de Desarrollo de la Escuela de Salud Pública, exponiendo su impacto en el quehacer académico y la gestión académica de la institución, y explicitando tanto los factores facilitadores como las dificultades encontrados en su ejecución y las estrategias implementadas para superar estas últimas.

En este artículo se describen los principales logros observados durante el periodo, se identifican los factores que facilitaron su ejecución, las limitaciones encontradas y las medidas adoptadas para superarlas.

\section{Formulación, diseño e implementación del PED}

El año 2000 se inició un ciclo de renovación de la ESP con la formulación y diseño del Plan Estratégico de Desarrollo "Un camino de renovación de la salud pública en la Universidad de Chile", utilizando una metodología de planificación participativa en la que colaboraron académicos, profesionales no académicos y representantes de los funcionarios administrativos.

La formulación del segundo PED, 2007-2011, igualmente realizado en forma integrada y conjunta por todos los estamentos de 
la ESP, contó con la asesoría de una empresa externa especializada en desarrollo institucional. El proceso fue guiado por el "Grupo Conductor" (GC) responsable de coordinar el diseño del plan, orientar la labor de las fuerzas de tarea, sistematizar la información obtenida de ellas y presentar los avances al "Grupo Amplio" (GA), constituido por los integrantes de los tres estamentos. Este proceso incluyó además la validación periódica de dichos avances de acuerdo con el siguiente esquema (figura 2):

$\mathrm{GC}=$ Grupo conductor
$\mathrm{GA}=$ Grupo amplio

DIAGNÓSTICO

GC

Análisis stakeholders

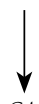

GA

Involucramiento y

Taller 1

Grupo amplio

elaboración diagnóstico

compartido

\section{PLANIFICACIÓN}

Programación y control

Capacidades, déficits y planes de acción

$\hat{1}$
$\vdots$
$\vdots$
GA

Visualización de un estado deseado
Taller 2

Grupo amplio

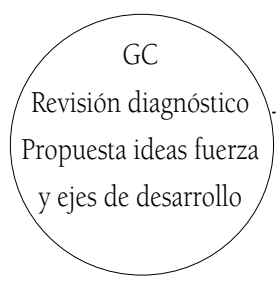

Se constituyeron equipos de trabajo en torno a tres ejes estratégicos: quehacer académico de excelencia; fortalecimiento de la relación con el entorno y organización moderna al servicio del quehacer académico. Para cada uno de los ejes se formularon iniciativas y se definieron metas, se designaron responsables y se establecieron hitos que permitieran monitorear los avances del plan, que fue validado en claustros periódicos. Como parte de este proceso se elaboró la misión y visión de la Escuela de Salud Pública. 
La definición y priorización de las líneas de desarrollo tuvo en cuenta los siguientes criterios:

- Consistencia con las ideas-fuerza definidas en la visión de la ESP.

- Coherencia con el Plan de Desarrollo Institucional de la Facultad de Medicina.

- Coherencia con las políticas de la Universidad de Chile.

- Capacidad de mover la organización en la dirección deseada.

- Factibilidad técnica y económica.

- Contribución al avance en la carrera académica y funcionaria de los miembros de la ESP.

- Contribución a la reducción de brechas existentes o potenciación de las fortalezas en relación con otras universidades.

Finalmente, se constituyeron siete equipos de trabajo que definieron los proyectos prioritarios estableciendo tareas específicas y metas tangibles (figura 3). El plan así diseñado fue aprobado en el Comité Académico de la escuela y luego validado por el claustro.

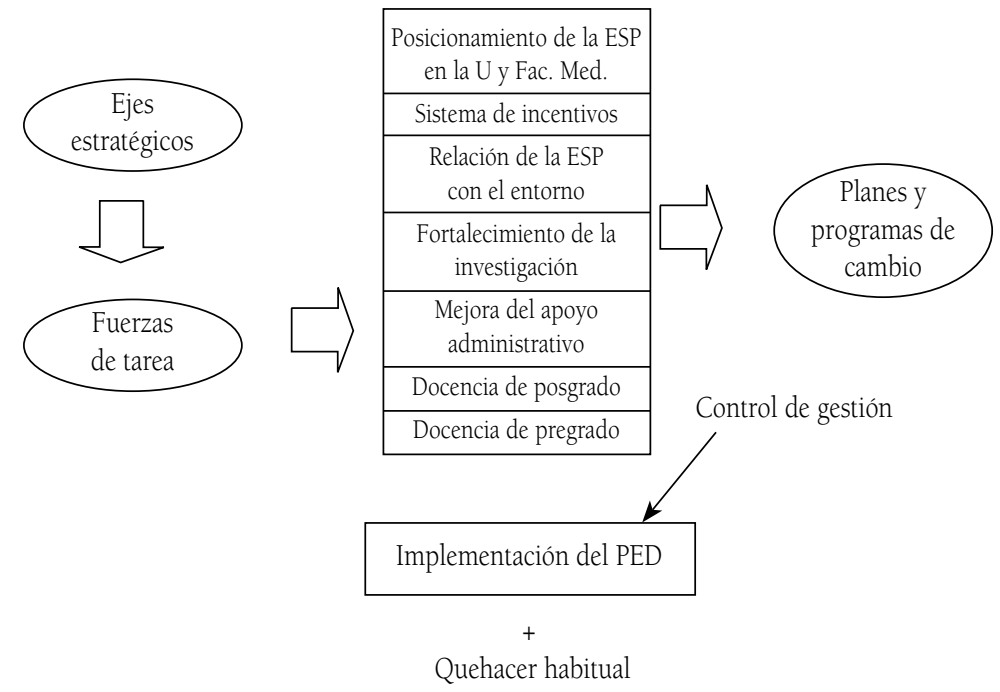

Plan estratégico de desarrollo 
Terminada la fase de diseño, la implementación se realizó bajo la lógica de proyectos para las diferentes iniciativas, incorporándose estos en la estructura regular de la escuela y recayendo la responsabilidad de su ejecución en la dirección e instancias institucionales correspondientes. Los avances en la implementación del plan fueron monitoreados en forma sistemática, informando periódicamente al claustro académico ampliado.

\section{Logros y limitaciones de la experiencia}

\section{Renovación y perfeccionamiento del cuerpo académico y profesional no académico}

A lo largo de los años se llevó a cabo una activa política de recursos humanos que ha tenido impacto en el desarrollo de nuestra escuela. Se otorgó prioridad a la contratación de nuevos académicos/as y a la formación doctoral de un número importante de ellos/as en el país y en el extranjero, política que ha comenzado a dar frutos. Igualmente se continuó con el fortalecimiento y profesionalización de las unidades de colaboración y apoyo al quehacer académico. Durante este periodo se materializó la creación de la Unidad de Tecnologías de la Información para la Educación (UTIE), la modernización de la biblioteca y la innovación en los productos de difusión de la oferta académica.

Ampliación y modernización de los programas de grados académicos

Lo más destacado en este periodo fue la creación del Programa de Doctorado en Salud Pública el año 2003, acreditado y beneficiario de dos proyectos Mecesup en los últimos siete años, con una demanda creciente y 12 graduados en los últimos tres años.

Hace tres años el magíster en Salud Pública, que se dicta desde el año 1981, experimentó cambios significativos, pasando de un formato presencial a uno semipresencial con mayor flexibilidad y personalización. Sin duda, estos cambios han tensionado los paradigmas tradicionales de docencia, transitando desde programas rígidos a programas de formato más flexible; desde actividades que se realizaban exclusivamente en el aula a una combinación de actividades 
presenciales y no presenciales, sincrónicas y diacrónicas; desde una enseñanza centrada en el docente a un proceso centrado en el alumno donde este es el actor principal en el desarrollo de sus competencias. Igualmente, el apoyo de la UTIE ha permitido incorporar el uso de modernas tecnologías en programas de posgrado, diplomas y cursos, logrando una mayor adecuación a las necesidades de profesionales que requieren formación en salud pública.

La docencia de pregrado es reconocida en la ESP como una responsabilidad ineludible y deseable, ya que permite influir en la salud de la población mediante la formación de nuevos profesionales, constituyendo un semillero de futuros especialistas en salud pública. Igualmente, la ESP ha tenido una activa participación en el Proyecto de Innovación Curricular de la Facultad de Medicina, lo cual ha generado requerimientos crecientes de habilitación de los docentes.

Apoyo a la investigación concursable y no concursable La investigación es uno de los pilares del quehacer de toda institución académica; sin embargo, durante la última década en nuestra escuela no se logró un desarrollo significativo en cuanto a su calidad y pertinencia. Esto puede explicarse en gran parte por: 1. la ausencia de un contingente suficiente de investigadores, ya que su formación a nivel doctoral solo comenzó en nuestro país el año 2003 y las oportunidades para estudiar en el extranjero han sido siempre limitadas. 2. la falta de tiempo protegido para realizar investigación, debido a que la docencia ocupa gran parte del tiempo contratado. 3. la dificultad para constituir equipos de investigación estables, aspecto relacionado con la corta duración de los proyectos de investigación e incertidumbre sobre la continuidad de ellos. 4. escaso financiamiento público de la investigación esencial y relevante en el campo de salud pública.

La estrategia implementada en la ESP para enfrentar esta situación incluyó la contratación de académicos jóvenes y la prioridad otorgada a su formación doctoral para un número importante de ellos/ as; el apoyo en la preparación de proyectos competitivos presentados a fondos de financiamiento concursables; el estímulo y apoyo a la presentación de trabajos en congresos científicos y su publicación en 
revistas indexadas. Como es sabido, los resultados de una política de investigación son observables en el largo plazo y en nuestra institución solo en los últimos años han comenzado a verse resultados. En síntesis, este objetivo estratégico solo se cumplió parcialmente.

Por otra parte, en este periodo la ESP desarrolló capacidades y competencias en la ejecución de estudios y proyectos de asistencia técnica a instituciones de gobierno, autónomas y privadas, asesorías que junto con contribuir al éxito de ellos permitieron la adquisición de nuevos conocimientos y experiencia del cuerpo académico de la escuela.

Fortalecimiento de las relaciones académicas nacionales e internacionales

En este tiempo las relaciones al interior de la universidad se ampliaron, incluyendo mayor interacción con otras facultades e institutos, e igualmente se fortaleció la relación con otras universidades nacionales.

En el ámbito internacional se suscribieron o renovaron 17 convenios de colaboración, lo que permitió contar con apoyo en la formación de posgrado, con la participación de académicos extranjeros en eventos científicos y la formulación conjunta de proyectos de investigación. Igualmente un número creciente de nuestros académicos y estudiantes de posgrado realizaron estadías en universidades de Norteamérica y Latinoamérica y europeas de excelencia.

A partir de 2009 se observó un salto cualitativo al ser invitados por universidades extranjeras a participar en proyectos de investigación y la constitución de redes. Destacan las experiencias con la Universidad de Ottawa, el Instituto Nacional de Salud Pública de México y las universidades de Helsinki y Tampere en Finlandia, entre otras.

\section{Relaciones con el sector público de salud}

En el transcurso de los años la relación con el sector salud no fue fácil ni fluida, aun cuando desde la ESP se realizaron numerosos esfuerzos 
por establecerlas. Entre los factores que influyeron en esta situación es posible identificar: 1. la frecuente rotación de los directivos del sector salud impidiendo contar con interlocutores estables; 2. la dificultad para reconocer, especialmente respecto del Ministerio, los aportes que puede hacer la academia en el diseño, implementación y evaluación de programas, en capacitación de personal y en realización de estudios. La estrategia para superar esta situación incluyó efectuar capacitaciones, diplomas y cursos en los servicios de salud y secretarías regionales ministeriales; fortalecer relaciones personales con responsables de unidades específicas del sector y participar en diversas comisiones ministeriales.

Presencia institucional frente a instituciones y públicos de su interés

La relación con la Organización Panamericana de la Salud ha sido la de mayor significación, participando en iniciativas conjuntas como el Campus Virtual en Salud Pública en América Latina, el curso internacional sobre recursos humanos en salud, el Programa Líderes en Salud Internacional, el Observatorio de Género y el Programa de Medicamentos e Innovación, entre otros. Dentro de una relación positiva se consideró necesario focalizar los esfuerzos en áreas prioritarias para la ESP y negociar en mejores términos esa cooperación.

Igualmente, durante este periodo se enfatizó el posicionamiento de la institución frente a sus públicos de interés; es así como progresivamente se fortaleció la presencia de la escuela en los medios de comunicación, especializados e institucionales, mediante columnas de opinión, entrevistas a académicos y comunicados de prensa, dando a conocer las propuestas y opiniones institucionales en temas de salud pública. Además, se amplió y perfeccionó la difusión de los programas académicos, encuentros científicos y eventos de extensión realizados por la ESP.

Mejoramiento de la gestión académica de la ESP

En este ámbito, la Subdirección de Administración y Finanzas cumplió un rol facilitador y de apoyo al quehacer académico. Con 
este fin se llevó a cabo un conjunto de iniciativas como actualización de su estructura y funcionamiento con el propósito de establecer y dar a conocer los roles y funciones de sus integrantes; elaboración de un manual de procedimientos y definición de indicadores que permitiesen evaluar el cumplimiento de metas y monitorear el proceso de mejoramiento continuo. Sin embargo, quedó pendiente implementar y poner a disposición de los académicos instrumentos que permitieran evaluar la calidad y oportunidad del apoyo administrativo a su labor académica.

\section{Aprendizajes de la experiencia}

En síntesis, entre los años 2000 y 2010 fue posible observar un desarrollo significativo en el ámbito académico, destacando el fortalecimiento del cuerpo académico y la profesionalización de la gestión académica, así como la formación avanzada de recursos humanos. La investigación constituyó un desafío cuyos resultados solo se conocerán a mediano plazo. La relación con el entorno se ha ampliado y profundizado, generando interesantes perspectivas de desarrollo futuro. Igualmente, se requiere un mayor impulso a las iniciativas que permitan mejorar el apoyo administrativo a la labor académica.

Por otra parte, la metodología de planificación utilizada, en la que el proceso se inició con la identificación de los stakeholders y las características del entorno, permitió el reposicionamiento de la Escuela de Salud Pública a nivel nacional e internacional. El que la Facultad de Medicina de la cual depende la escuela haya estado desarrollando al mismo tiempo su plan estratégico, constituyó una ventajosa sinergia para el desarrollo de ambos organismos. Igualmente, el PED se demostró útil por su aporte a una adaptación oportuna de la ESP a las condiciones del entorno.

Como herramienta de apoyo a la dirección de la escuela, el PED contribuyó a priorizar aquellas acciones más relevantes para el desarrollo armónico de la institución, alineando los esfuerzos de sus integrantes en torno a los ejes e iniciativas construidos participativamente, constituyéndose en una herramienta útil para el cumplimiento de los compromisos de gestión. 
Es posible identificar dos elementos clave en el éxito del proceso: la participación activa de los integrantes de la organización y el monitoreo del plan. En cuanto a participación, nuestra buena experiencia presentó altibajos en el tiempo, requiriendo actividades motivacionales para mantenerla. El seguimiento del plan estuvo a cargo de un "controlador" que en forma permanente comparaba los resultados esperados con los observados, e informaba al Comité Académico sobre el grado de avance de cada una de las iniciativas; lo que permitía analizar los motivos del mayor o menor avance y promover acciones para un mejor desempeño. Igualmente, en forma periódica se informaba los resultados de las evaluaciones al claustro, lo cual permitía retroalimentar a los responsables de cada una de las iniciativas. Sin embargo, cabe reconocer que en la medida en que el proceso avanzaba se requirió un mayor esfuerzo para estimular las evaluaciones participativas.

En conclusión, consideramos que la ejecución de este plan estratégico permitió que la ESP, en un periodo de 10 años y más allá de su quehacer académico, hiciera un efectivo aporte a la gestión académica, contribuyendo al mejoramiento del nivel de salud y calidad de vida de la población chilena. En síntesis, una contribución a la construcción de la salud pública del futuro.

Recibido: 26/05/2011

Aceptado: 26/10/2011 